\title{
Occult HBV infection may negatively impact on drug survival in patients with rheumatoid arthritis on treatment with a first biologic drug. An appraisal from the Biologic Apulian Registry (BIOPURE)
}

\author{
G. Carlino1, M. Fornaro², L. Santo ${ }^{3}$, R. Bucci ${ }^{4}$, A. Semeraro ${ }^{5}$, L. Quarta ${ }^{6}$, \\ F. D’Onofrio ${ }^{7}$, A. Marsico ${ }^{5}$, C. Zuccaro ${ }^{8}$, P.C. Falappone ${ }^{8}$, D. Mazzotta 8 , \\ F.P. Cantatore ${ }^{7}$, M. Muratore ${ }^{6}$, F. lannone ${ }^{2}$ \\ ${ }^{1}$ Rheumatology Service, ASL LE - DSS Casarano and Gallipoli (LE), Italy; ${ }^{2}$ Department of Emergency \\ and Organ Transplantation-Rheumatology Unit, University of Bari, Italy; ${ }^{3}$ Unit of Rheumatology, ASL BT, Italy; \\ ${ }^{4}$ Rheumatology Hospital Unit, A.O.U. Foggia, Italy; ${ }^{5}$ Unit of Rheumatology, ASL Taranto, Italy; \\ ${ }^{6}$ Unit of Rheumatology, "V. Fazzi" Hospital, Lecce, Italy; 'Unit of Rheumatology, University-Hospital \\ of Foggia, Italy; ${ }^{8}$ Ambulatory of Rheumatology, Hospital of Brindisi, Italy
}

\section{SUMMARY}

We performed a retrospective analysis to evaluate the survival on first line biologic drug of rheumatoid arthritis (RA) patients with potential occult $\mathrm{HBV}$ infection (pOBI).

We analysed longitudinal data of 486 consecutive RA patients starting a first biological drug in a time frame from $1^{\text {st }}$ January 2008 to $31^{\text {st }}$ December 2014. Demographic and disease related characteristics were collected at baseline and at the last observation visit. Baseline serological markers of $\mathrm{HBV}$ infection and causes of treatment discontinuation were also recorded. Primary endpoint was the influence of pOBI on drug survival, estimated by Kaplan-Meier life table analysis. Estimates hazard ratios (HRs) of drug discontinuation, adjusted for disease characteristics, biological drug class and $\mathrm{HBcAb}$ status were computed by Cox-regression models.

The retention rate was significantly lower in pOBI positive patients $(58.2 \%)$ when compared to pOBI negative ones $(67.8 \%)$ and this data was confirmed also when only discontinuation due to ineffectiveness was considered (pOBI positive $66.4 \%$ vs pOBI negative $75.3 \%$, long rank 7.93, $\mathrm{p}=0.005$ ). Cox regression models showed a significant association between HBcAb-neg (HR 0.58, 0.41-0.84), higher ESR-DAS28 at baseline (HR 1.07, 1.03-1.11) or RF/ACPA-neg (HR 1.46, 1.04-2.06) and drug discontinuation.

Occult HBV infection seems to influence negatively the effectiveness of biological therapies in RA patients.

Key words: Drug survival; Occult HBV infection; Predictors; safety; Rheumatoid arthritis.

Reumatismo, 2019; 71 (1): 24-30

\section{INTRODUCTION}

Towadays biologic therapies play a pivotal role in the management of patients with rheumatoid arthritis (RA) and according to current EULAR recommendations seven biologic drugs are approved for administration to RA patients who are inadequate responders to methotrexate (1): five tumour necrosis factor alpha (TNF- $\alpha$ ) inhibitors (infliximab, etanercept, adalimumab, golimumab, certolizumab), one humanized monoclonal antibody against interleukin 6 (IL-6) receptor (tocilizumab), and one soluble CTLA4-Fc fusion protein (abatacept). The immunosuppressive effects of these drugs made it mandatory to screen the patients for possible reactivation of latent infections, such as hepatitis $\mathrm{B}$ virus $(\mathrm{HBV})(2,3)$. In the haematological field, due to high risk of HBV reactivation, great emphasis was given to patients with occult HBV infection (OBI), defined by the presence of HBV-DNA in the liver associated with the absence of hepatitis B surface antigen (HBsAg) in serum. Since liver biopsy is not routinely performed, potential OBI (pOBI) may be suspected when 
hepatitis B core antibody ( $\mathrm{HBcAb})$, with or without hepatitis B surface antibody ( $\mathrm{Hb}$ $\mathrm{sAb}$ ) and without HBV DNA overt replication, is detected (4). However, some studies downgraded these concerns by demonstrating a good safety profile of biologic DMARDs (bDMARDs) in OBI patients treated for rheumatic diseases $(5,6)$. Recently, this issue was confirmed by the Italian consensus Guidelines for the management of $H B V$ infections in patients with $R A$, which encourages monitoring of HbsAg in RA patients treated with biologic therapies, without need for universal prophylaxis to prevent HBV reactivation (3). However, whether the status of occult HBV infection (OBI) may influence the clinical response to bDMARDs in RA patients has never been investigated. In real-world settings, a benchmark for the effectiveness and safety of a treatment is the time of its persistence on therapy. The aim of this study was to evaluate whether pOBI should impact the drug survival on first line bDMARD of RA patients from Biologic Apulian Registry (BIOPURE).

\section{MATERIALS AND METHODS}

This was a retrospective multicentric observational study collecting data from patients affected with RA treated with biologic therapies from 9 Apulian rheumatologic centres and recorded in the BIOPURE. Data from 486 consecutive RA patients starting a first biological drug in a time frame from $1^{\text {st }}$ January 2008 to $31^{\text {st }}$ December 2014 were analysed. All patients fulfilled 1987 and 2010 classification criteria for RA $(7,8)$. pOBI was defined as the presence of $\mathrm{HBcAb}$ along with the absence of HBsAg and HBV DNA replication. At baseline, the following demographics and clinical characteristics were recorded: age, gender, presence of comorbidities, rheumatoid factor (RF) and anti-citrullinated peptide antibody (ACPA) positivity, duration of disease, Health Assessment Questionnaire disability index (HAQ-DI) as measure of functional disability, concomitant therapy with glucocorticoids (prednisone equivalent) or methotrexate (MTX), and type of biologic (tocilizumab, abatacept, etanercept and TNF- $\alpha$ monoclonal antibody including Infliximab, adalimumab, golimumab and certolizumab). As composite indexes of clinical disease activity, the ESR-disease activity Score on 28 joints (ESR-DAS28) and clinical disease activity index (CDAI) were assessed at baseline and at the last observation. Furthermore, baseline serological markers of HBV infection, including $\mathrm{HBsAg}, \mathrm{HBsAb}, \mathrm{HBcAb}$ and HBV-DNA levels were also acquired. Patients with concomitant hepatitis $C$ virus infection were excluded.

Baseline demographics and disease characteristics were evaluated using standard descriptive statistics. Categorical data were expressed as a percentage and continuous variables as mean \pm 1 standard deviation (SD). Comparisons between pOBI patients and non-pOBI patients were carried out by chi-square test or analysis of variance (ANOVA), where appropriate. Patients not attending follow-up or patients who stopped therapy due to reasons deemed unrelated to therapy (pregnancy, relocation, clinical remission, or other) were removed from the study. The time to discontinuation was defined as the time between biologic initiation and last administration plus dispensation interval. Crude drug retention rate and mean survival time (MST) were estimated using Kaplan-Meier (K-M) life-table method and differences between groups were compared using the long-rank test. Estimated hazard ratios (HRs) of discontinuing bDMARD were assessed by performing a multivariate stepwise backward Cox regression analysis. The variables included in the analysis were: age, gender, co-therapy with MTX or steroids at baseline, HAQ, CDAI, type of biologics and $\mathrm{HBcAb}$ status. A p value $<0.05$ was considered statistically significant. To analyse the data, IBM SPSS statistics 21 was used.

\section{RESULTS}

We studied a total of 486 patients who started a first biologic drug due to active RA despite adequate treatment with conventional 
Table I - Demographic and clinical characteristics of Rheumatoid arthritis (RA) patients at baseline according the presence of potential occult HBV infection (pOBI). Comparisons between variables of $\mathrm{pOBI}$ neg (HBcAb neg) and $\mathrm{pOBI}$ pos ( $\mathrm{HBcAb}$ pos) patients were expressed in $p$ values. A $p$ value $<0.05$ was considered statistically significant.

\begin{tabular}{|l|c|c|c|c|}
\hline & $\begin{array}{c}\text { All } \\
\text { (no. 486) }\end{array}$ & $\begin{array}{c}\text { HBcAb neg } \\
\text { (no. 376) }\end{array}$ & $\begin{array}{c}\text { HBcAb pos } \\
\text { (no. 110) }\end{array}$ & p \\
\hline Age & $57 \pm 11$ & $58 \pm 11$ & $57 \pm 12$ & ns \\
\hline Female no (\%) & $379(78)$ & $295(78.5)$ & $84(76.4)$ & ns \\
\hline Disease duration & $46 \pm 65$ & $44 \pm 66$ & $54 \pm 61$ & ns \\
\hline Comorbidities no (\%) & $205(42.2)$ & $160(42.6)$ & $45(40.9)$ & ns \\
\hline ESR-DAS28 & $4.6 \pm 2.4$ & $4.6 \pm 2.6$ & $4.7 \pm 1.2$ & ns \\
\hline CDAl & $20.1 \pm 11$ & $20 \pm 11$ & $20 \pm 11$ & ns \\
\hline HAQ-DI & $1.3 \pm 0.7$ & $1.3 \pm 0.7$ & $1.2 \pm 0.7$ & ns \\
\hline RF/ACPA no (\%) & $352(73)$ & $270(72)$ & $82(76)$ & ns \\
\hline Etanercept no (\%) & $164(34)$ & $136(36)$ & $28(26)$ & ns \\
\hline TNFa mAb no (\%) & $147(32)$ & $121(32)$ & $26(24)$ & ns \\
\hline Tocilizumab no (\%) & $79(16)$ & $52(14)$ & $27(24)$ & $<0.05$ \\
\hline Abatacept no (\%) & $96(20)$ & $67(18)$ & $29(26)$ & $<0.05$ \\
\hline Glucocorticoids no (\%) & $377(78)$ & $290(77)$ & $87(79)$ & ns \\
\hline MTX no (\%) & $403(83)$ & $323(85)$ & $80(72)$ & $<0.05$ \\
\hline MTX mg/weekly & $12.9 \pm 2.8$ & $13 \pm 2.8$ & $12.8 \pm 2.9$ & ns \\
\hline
\end{tabular}

Values are the mean 1 SD unless otherwise indicated. ESR-DAS28, erythrocyte sedimentation rate-28 joints Disease Activity Score; CDAI, Clinical Disease Activity Index; HAQ, Health assessment questionnaire disability index; RF, Rheumatoid Factor; ACPA, anti-citrullinated protein antibody; TNF $\alpha \mathrm{mAb}$, Tumour Necrosis Factor $\alpha$ monoclonal antibody (Infliximab, Adalimumab, Golimumab, Certolizumab); MTX, Methotrexate.

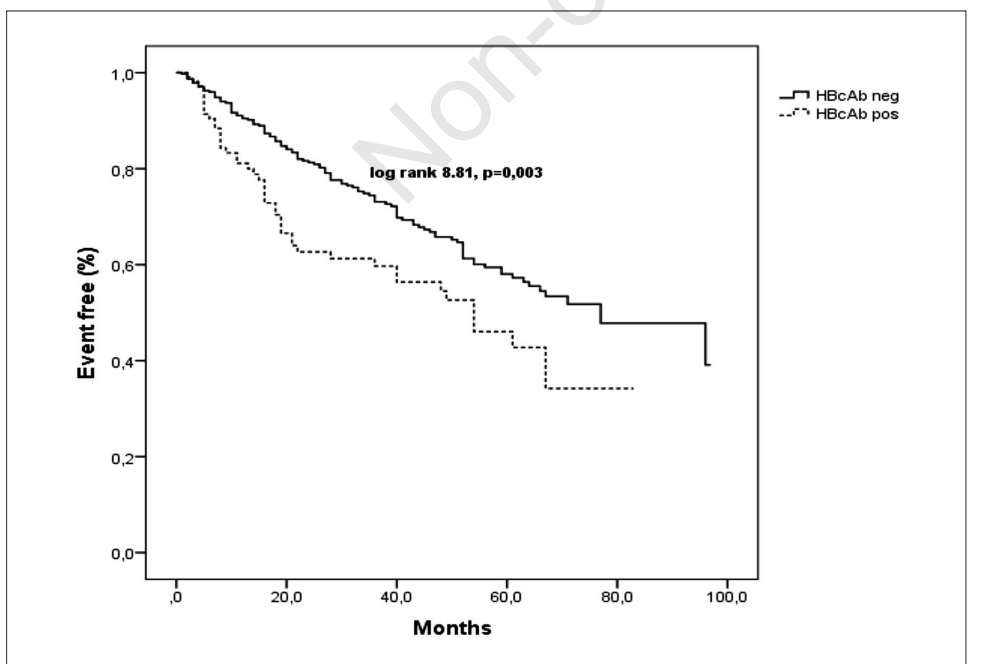

Figure 1 - Survival curve analysis of rheumatoid arthritis patients treated with biologics classified according presence of potential HBV infection (pOBI). Drug survival assessed by Kaplan-Meier life-table method and comparison among pOBI neg (HBcAb neg) and pOBI pos (HBcAb pos) patients.
DMARDs. Of these, $110(22.6 \%)$ patients had pOBI, while $376(77.4 \%)$ patients were negative. No patients underwent antiviral therapy for prophylaxis. Comprehensive demographics and baseline clinical data of the two cohorts are shown in Table I. There were no statistically significant differences between the two groups for most of the data, except for frequency of Tocilizumab and Abatacept that were significantly more prescribed as first bDMARD in pOBI positive RA patients $(\mathrm{p}<0.05)$. On the other hand, MTX was significantly less given in pOBI positive RA patients $(\mathrm{p}<0.05)$.

Crude drug survival was estimated by $\mathrm{K}-\mathrm{M}$ curves and the global retention rate was $65,6 \%$ at end of follow-up, the mean survival time being $(\mathrm{MST})=62.8$ (95\% CI: 59-67) months. Comparing the drug survival between groups according to $\mathrm{HBcAb}$ serology, the retention rate was significantly lower for pOBI positive RA patients $(58.2 \% \quad(\mathrm{MST}=48.2 \quad(95 \%$ CI: 41-55) months) than pOBI negative ones $[67.8 \%$ (MST=65.5 (95\% CI: 61-70) months] (long rank 8.81, $\mathrm{p}=0.003$ ) (Fig. 1). Likewise, the drug persistence was still significantly lower in pOBI positive RA when discontinuation as a result of ineffectiveness was considered, as it was $66.4 \%$ [MST $=53.4$ (95\% CI: 46-60) months] for pOBI positive and $75.3 \%$ [MST $=71(95 \%$ CI: 66-75) months] for pOBI negative RA patients (long rank 7.93, $\mathrm{p}=0.005$ ) (Fig. 2). Drug discontinuation as a result of adverse events was similar in pOBI positive or negative RA patients (long rank 1.6, $p=0.2$ ), however. These findings are also confirmed in a sub-analysis including patients treated with TNF $\alpha$ inhibitors (TNFi) and considering ineffectiveness as cause of discontinuation, drug persistence being lower in pOBI positive patients [53.7\% MST $=45.2$ (95\% CI: 35-54) months] than pOBI negative ones [73\% MST $=72.1$ (95\% CI: $67-$ 76) months] (long rank 17.8, $\mathrm{p}=0.001$ ). Drug discontinuation as a result of adverse events in patients treated with TNFi, comparing pOBI status, was similar (long rank $0.63, p=0.42$ ), on the other hand. Stepwise backward multiple Cox-regression analysis was then used to search for possible predic- 
tors of discontinuation in the whole cohort. ACPA/RF negative RA patients ( $\mathrm{HR}=1.46$, 95\% CI: 1.04-2.06) and patients with higher ESR-DAS28 at baseline (HR=1.07, 95\% CI: 1.03-1.11) had higher hazards in stopping the therapy, while $\mathrm{HbcAb}$ negative patients had lower hazards $(\mathrm{HR}=0.58,95 \%$ CI: 0.41-0.84) in discontinuing the treatment (Table II). Others baseline factors, such as age, gender, co-therapy with MTX or steroids, HAQ, CDAI, type of biologics were not correlated with drug discontinuation. In the whole observation period there were no reactivations of Hepatitis $B$ virus infection in pOBI patients.

Biologics therapy was safe and well tolerated. Of 179 (36.8\%) patients stopping treatment, only $36(7.4 \%)$ withdrew due to adverse events, $130(26.7 \%)$ due to ineffectiveness and $13(2.7 \%)$ due to other causes. Finally, two patients stopped bDMARD therapy on account of persistent clinical remission in the pOBI negative group (1 tocilizumab, 1 etanercept) and none in the pOBI positive group. These patients continued MTX monotherapy.

\section{DISCUSSION AND CONCLUSIONS}

HBV infection affects nearly 278 million people worldwide (9). In our cohort we found a prevalence of $22.6 \%$ pOBI, which can be considered high. Currently, the exact prevalence of $\mathrm{HBcAb}$ positivity in the Puglia region is unknown, but other studies in southern Italy, regarding patients with Non-Hodgkin Lymphoma or psoriasis (10, 11), have reported similar high prevalence of pOBI. Moreover, the old age of our cohort may have selected patients with prior

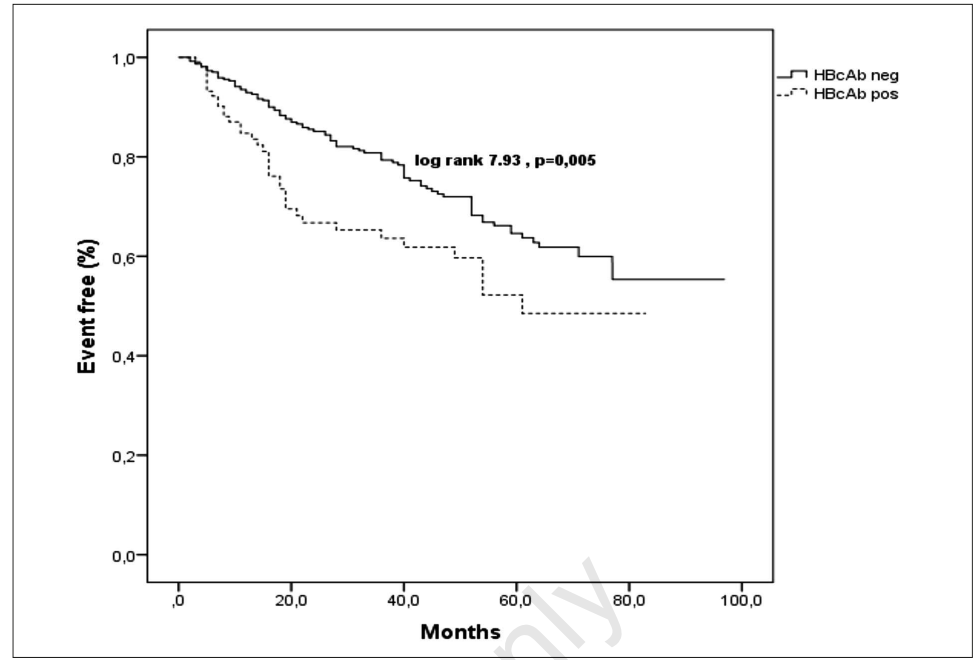

Figure 2 - Survival curve analysis of Rheumatoid arthritis patients suspending biologic for ineffectiveness classified according presence of potential HBV infection (pOBI). Drug survival assessed by KaplanMeier life-table method and comparison among pOBI neg (HBcAb neg) and $\mathrm{pOBI}$ pos (HBcAb pos) patients.

higher risk of HBV infection. For this reason, in our daily practice it is frequent to come across patients with positive HBV serology, and some concerns for reactivation of OBI may rise when a bDMARD treatment is commenced. However, numerous studies have shown a good safety profile for various bDMARD drugs in RA patients with OBI $(5,6,12-14)$ and global prophylaxis is not recommended for these patients. Only OBI patients treated with Rituximab seem to have a higher risk of HBV reactivations (15), but this drug is not currently approved in first line therapy for RA and it was not considered in our study. A good safety profile has been also confirmed among our patients. We found 9 pOBI patients who stopped therapy on ac-

Table II - Final model of stepwise backward multivariate cox proportional-hazard regression of factors associated with bDMARD discontinuation.

\begin{tabular}{|l|c|c|c|}
\hline & HRs & $95 \% \mathrm{Cl}$ & p-value \\
\hline ESR-DAS28 & 1.07 & $1.035-1.116$ & 0.0001 \\
\hline RF/ACPA (neg/pos) & 1.46 & $1.041-2.065$ & 0.028 \\
\hline HBcAb (neg/pos) & 0.58 & $0.410-0.845$ & 0.004 \\
\hline
\end{tabular}

$\mathrm{HR}$, hazard ratio; $95 \% \mathrm{CI}$, confidence interval of hazard ratio; ESR-DAS28, erythrocyte sedimentation rate28 joints disease activity score. 
count of an adverse event and nobody had HBV reactivation as cause of discontinuation.

However, the aim of our study was different from other studies involving OBI patients. We wished to evaluate whether exposure to OBI might influence the effectiveness of first bDMARD therapy in RA patients, and we showed that pOBI positive RA patients had a significantly less drug survival rate than pOBI negative RA ones. Surprisingly, this was not due to an increase in adverse events, but to an impairment in clinical response to drug survival by effectiveness, which definitely decreased in pOBI positive RA patients. Baseline OBI status can also predict the persistence of treatment on first bDMARD as multivariate Cox regression analysis showed that pOBI negative RA patients had $42 \%$ less chance of discontinuing the therapy. Independently, RF/ ACPA negativity and higher ESR-DAS28 values were positively associated with $\mathrm{bD}$ MARD discontinuation.

To our knowledge, there are no studies in the literature that evaluate the status of $\mathrm{OBI}$ as a possible predictor of drug discontinuation. HBV DNA detection in the liver with concomitant absence of serum HBsAg is defined as OBI (4), which is not a silent status and the host's immunological factors are involved in the immune surveillance to inhibit HBV reactivation (16). The main players in controlling infections are CD4 and CD8 lymphocytes, which persist for several years after primary infection resolution as memory cells. An explanation of OBI can be given by the virus's ability, having entered the liver, to synthesize small amounts of antigens able to maintain a specific anti-HBV response over time (17). TNF $\alpha$ seems to play a primary role during HBV infection, inhibiting viral replication as shown in mice models (18). Also IL-6 may play a pivotal role during HBV infection, although literature studies are contradictory. IL- 6 production is certainly upregulated in patients with chronic HBV infection $(19,20)$, but while some studies showed how IL-6 can suppress HBV replication in the HBV replicating cell line (21), other studies underlined its possible role in HBV related hepatocarcinogenesis (22, 23). The relation between HBV infection and CTLA-4 is less known. Only one experimental study highlighted that CTLA-4 reduction by RNA interference mechanism in chronic HBV patients led to INF- $\gamma$ secretions via TH1/TH2 response (24). In this complex scenario, in which numerous cytokines and cellular act, we cannot rule out the possibility that OBI patients may experience an impaired clinical response due to this OBI-related immune activation state.

Interestingly, OBI status is not the only factor that might influence the clinical response to bDMARD without a plausible reason. Indeed, this is also the case with smoking, and large studies have shown that smoking predicted TNF inhibitor discontinuation by ineffectiveness, even though the true underlying mechanism was not clarified (25-29). Likewise, female gender is another example of an unknown confounding factor associated with poor outcome and bDMARD discontinuation in RA population $(25,28,30)$. Consistently with the literature, our study confirms that seronegative RA and patients with higher ESR-DAS28 at baseline have higher risk of discontinuing bDMARDs (31-33).

It is worth noting that pOBI patients were more frequently treated with non-TNFi, suggesting that rheumatologists should show some caution when dealing with OBI RA patients. Tocilizumab and Abatacept are deemed to have a safer profile in OBI patients and perhaps this may influence clinician's choices $(5,13,34,35)$. Also MTX was less frequently given to pOBI RA patients suggesting that, even though numerous studies and recommendations indicate a positive safety profile for MTX (3, 15, 36), rheumatologists should still be cautious when dealing with these patients. However, multivariate Cox regression analysis did not show an association between MTX intake and drug survival for our patients.

Some drawbacks of our study need to be underlined. This was a multi-centre retrospective analysis of longitudinal data and some unknown biases may have been left out. Furthermore, we did not analyse the 
outcome of the single bDMARD because of the low number of patients on treatment with each drug.

In conclusion, our study provides preliminary evidence that $\mathrm{pOBI}$ status may predict a poor clinical outcome in naïve-biologic RA patients starting a bDMARD in reallife settings. The reason for these findings remains to be discovered. Further studies on larger cohorts will be needed to confirm these data, to assess a possible specific bDMARD related effect, and to decipher its possible biological significance.

Contributions: GC, MF, contributed equally to this work.

\section{REFERENCES}

1. Smolen JS, Landewé R, Bijlsma J, et al. EULAR recommendations for the management of rheumatoid arthritis with synthetic and biological disease-modifying antirheumatic drugs: 2016 update. Ann Rheum Dis. 2017; 76: 960-77.

2. Lampertico P, Agarwal K, Berg T, et al. EASL 2017 Clinical Practice Guidelines on the management of hepatitis B virus infection. J Hepatol. 2017; 67: 370-98.

3. Sebastiani M, Atzeni F, Milazzo L, et al. Italian consensus Guidelines for the management of hepatitis B virus infections in patients with rheumatoid arthritis. Joint Bone Spine. 2017; 84: 525-30.

4. Raimondo G, Allain JP, Brunetto MR, et al. Statements from the Taormina expert meeting on occult hepatitis B virus infection. J Hepatol. 2008; 49: 652-7.

5. Barone M, Notarnicola A, Lopalco G, et al. Safety of long-term biologic therapy in rheumatologic patients with a previously resolved hepatitis B viral infection. Hepatology. 2015; 62: 40-6.

6. Giannitti C, Lopalco G, Vitale A, et al. Longterm safety of anti-TNF agents on the liver of patients with spondyloarthritis and potential occult hepatitis B viral infection: an observational multicentre study. Clin Exp Rheumatol. 2017; 35: 93-7.

7. Aletaha D, Neogi T, Silman AJ, et al. 2010 Rheumatoid arthritis classification criteria: an American College of Rheumatology/European League Against Rheumatism collaborative initiative. Arthritis Rheum. 2010; 62: 2569-81.

8. Arnett FC, Edworthy SM, Bloch DA, et al. The American Rheumatism Association 1987 revised criteria for the classification of rheumatoid arthritis. Arthritis Rheum. 1988; 31: 315-24.
9. Schweitzer A, Horn J, Mikolajczyk RT, et al. Estimations of worldwide prevalence of chronic hepatitis B virus infection: a systematic review of data published between 1965 and 2013. Lancet. 2015; 386: 1546-55.

10. Masarone M, De Renzo A, La Mura V, et al. Management of the HBV reactivation in isolated $\mathrm{HBcAb}$ positive patients affected with non hodgkin lymphoma. BMC Gastroenterol. 2014; 14: 31 .

11. Morisco F, Guarino M, La Bella S, et al. Lack of evidence of viral reactivation in $\mathrm{HBsAg-}$ negative $\mathrm{HBcAb}$-positive and $\mathrm{HCV}$ patients undergoing immunosuppressive therapy for psoriasis. BMC Gastroenterol. 2014; 14: 214.

12. Cantini F, Boccia S, Goletti D, et al. HBV reactivation in patients treated with antitumor necrosis factor-alpha (TNF- $\alpha$ ) agents for rheumatic and dermatologic conditions: a systematic review and meta-analysis. Int J Rheumatol. 2014; 2014: 926836.

13. Padovan M, Filippini M, Tincani A, et al. Safety of abatacept in rheumatoid arthritis with serologic evidence of past or present hepatitis B virus infection. Arthritis Care Res (Hoboken). 2016; 68: 738-43.

14. Nard FD, Todoerti M, Grosso V, et al. Risk of hepatitis B virus reactivation in rheumatoid arthritis patients undergoing biologic treatment: Extending perspective from old to newer drugs. World J Hepatol. 2015; 7: 344-61.

15. Marzano A, Bruno R, Fagiuoli S, et al. Gestione clinica dell'epatite B negli Immunocompromessi: aggiornamento italiano 2017 (AISF). Associazione Italiana per lo studio del fegato; Feb 2017. Available from: www. webaisf.org/media/37755/gestione_clinica_ della_epatite_b_negli_immunocompromessi.2017.pdf

16. Raimondo G, Caccamo G, Filomia R, et al. Occult HBV infection. Semin Immunopathol. 2013; 35: 39-52.

17. Penna A, Artini M, Cavalli A, et al. Long-lasting memory $\mathrm{T}$ cell responses following selflimited acute hepatitis B. J Clin Invest. 1996; 98: 1185-94.

18. Tzeng HT, Tsai HF, Chyuan IT, et al. Tumor necrosis factor-alpha induced by hepatitis B virus core mediating the immune response for hepatitis B viral clearance in mice model. PLoS One. 2014; 9: e103008.

19. Zhang F, Yao S, Zhang M, et al. Roles of circulating soluble interleukin (IL)-6 receptor and IL-6 receptor expression on CD4+ T cells in patients with chronic hepatitis B. Int J Infect Dis. 2011; 15: e267-71.

20. Hösel M, Quasdorff M, Wiegmann K, et al. Not interferon, but interleukin-6 controls early gene expression in hepatitis B virus infection. Hepatology. 2009; 50: 1773-82.

21. Kuo TM, Hu CP, Chen YL, et al. HBV replica- 
tion is significantly reduced by IL-6. J Biomed Sci. 2009; 16: 41.

22. Li CH, Xu F, Chow S, et al. Hepatitis B virus $\mathrm{X}$ protein promotes hepatocellular carcinoma transformation through interleukin-6 activation of microRNA-21 expression. Eur J Cancer. 2014; 50: 2560-9.

23. Prieto J. Inflammation, HCC and sex: IL-6 in the centre of the triangle. J Hepatol. 2008; 48: 380-1.

24. Yu Y, Wu H, Tang Z, Zang G. CTLA4 Silencing with siRNA Promotes Deviation of Th1/ Th2 in Chronic Hepatitis B Patients. Cell Mol Immunol. 2009; 6: 123-7.

25. Plant D, Bowes J, Potter C, et al. Predictors of response to anti-TNF-therapy among patients with rheumatoid arthritis: results from the British Society for Rheumatology Biologics Register. Arthritis Rheum. 2011; 63: 645-53.

26. Cuppen BV, Jacobs JW, Ter Borg EJ, et al. Necessity of TNF-alpha inhibitor discontinuation in rheumatoid arthritis is predicted by smoking and number of previously used biological DMARD. Clin Exp Rheumatol. 2017; 35: 221-8.

27. Mattey DL, Dawes PT, Hassell AB, et al. Effect of psychological distress on continuation of anti-tumor necrosis factor therapy in patients with rheumatoid arthritis. J Rheumatol. 2010; 37: 2021-4.

28. McWilliams DF, Walsh DA. Factors predicting pain and early discontinuation of tumour necrosis factor- $\alpha$-inhibitors in people with rheumatoid arthritis: results from the British society for rheumatology biologics register. BMC Musculoskelet Disord. 2016; 17: 337.

29. Ramiro S, Landewé R, van der Heijde D, et al. Discontinuation rates of biologics in patients with rheumatoid arthritis: are TNF inhibitors different from non-TNF inhibitors? RMD Open. 2015; 1: e000155.

30. Souto A, Maneiro JR, Gómez-Reino JJ. Rate of discontinuation and drug survival of biologic therapies in rheumatoid arthritis: a systematic review and meta-analysis of drug registries and health care database. Rheumatology (Oxford). 2016; 55: 523-34.

31. Iannone F, Courvoisier DS, Gottenberg JE, et al. Body mass does not impact the clinical response to intravenous abatacept in patients with rheumatoid arthritis. Analysis from the "PanEuropean registry collaboration for abatacept (PANABA). Clin Rheumatol. 2017; 36: 773-9.

32. Thomas K, Flouri I, Repa A, et al. High 3-year golimumab survival in patients with rheumatoid arthritis, ankylosing spondylitis and psoriatic arthritis: real world data from 328 patients. Clin Exp Rheumatol. 2017 [Epub ahead of print].

33. Strand V, Miller P, Williams SA, et al. Discontinuation of biologic therapy in rheumatoid arthritis: analysis from the Corrona RA Registry. Rheumatol Ther. 2017; 4: 489-502.

34. Nagashima T, Minota S. Long-term tocilizumab therapy in a patient with rheumatoid arthritis and chronic hepatitis B. Rheumatology (Oxford). 2008; 47: 1838-40.

35. Tsuboi H, Tsujii A, Nampei A, et al. A patient with rheumatoid arthritis treated with tocilizumab together with lamivudine prophylaxis after remission of infliximab reactivated hepatitis B. Mod Rheumatol 2011; 21: 701-5.

36. Reddy KR, Beavers KL, Hammond SP, et al. American Gastroenterological Association Institute guideline on the prevention and treatment of hepatitis $\mathrm{B}$ virus reactivation during immunosuppressive drug therapy. Gastroenterology. 2015; 148: 215-9; quiz e16-7. 